\title{
Computational synthesis of 2D materials grown by chemical vapor deposition
}

\author{
Kasra Momeni ${ }^{1, a)} \mathbb{D}$, Yanzhou $\mathrm{Ji}^{2}$, Long-Qing Chen ${ }^{2}$ \\ ${ }^{1}$ Department of Mechanical Engineering, The University of Alabama, Tuscaloosa, AL 35487, USA \\ ${ }^{2}$ Materials Research Institute, Pennsylvania State University, University Park, PA 16802, USA \\ a) Address all correspondence to this author. e-mail: kmomeni@ua.edu \\ Received: 28 May 2021; accepted: 10 September 2021; published online: 12 October 2021
}

The exotic properties of 2D materials made them ideal candidates for applications in quantum computing, flexible electronics, and energy technologies. A major barrier to their adaptation for industrial applications is their controllable and reproducible growth at a large scale. A significant effort has been devoted to the chemical vapor deposition (CVD) growth of wafer-scale highly crystalline monolayer materials through exhaustive trial-and-error experimentations. However, major challenges remain as the final morphology and growth quality of the 2D materials may significantly change upon subtle variation in growth conditions. Here, we introduced a multiscale/multiphysics model based on coupling continuum fluid mechanics and phase-field models for CVD growth of 2D materials. It connects the macroscale experimentally controllable parameters, such as inlet velocity and temperature, and mesoscale growth parameters such as surface diffusion and deposition rates, to morphology of the as-grown $2 \mathrm{D}$ materials. We considered $\mathrm{WSe}_{2}$ as our model material and established a relationship between the macroscale growth parameters and the growth coverage. Our model can guide the CVD growth of monolayer materials and paves the way to their synthesis-by-design.

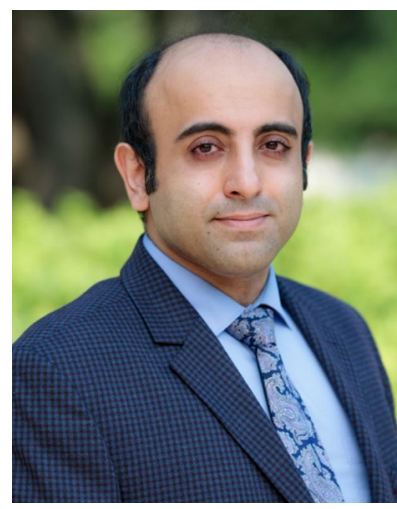

Kasra Momeni is an Associate Professor in the Mechanical Engineering Department at the University of Alabama. He was an Assistant Professor at Louisiana Tech University (2016-2020) and a Research Associate at the Pennsylvania State University (2015-2016) before joining the University of Alabama. He received a Ph.D. in Engineering Mechanics from Iowa State University in 2015, his MSc from Sharif University in 2008, and his B.S. in Mechanical Engineering in 2006 from KNT University. He obtained several awards, including the NSF-CAREER award, the Louisiana Tech National Recognition Award, and Iowa State University Research and Teaching Excellence Awards. His research interests include computational mechanics and material science, material genomics, hierarchical materials, and synthesis-by-design of materials.

\section{Introduction}

Two-dimensional (2D), also called single (or mono-) layer, materials are crystalline materials of only a single layer of atom thick. Their unique quantum properties are critical for technological applications in topographical computing, quantum informatics, and optoelectronic devices that have recently attracted the research community's attention. Furthermore, the 2D stacked van der Waals heterostructure materials are becoming a prime candidate for quantum material design. In addition to traditional approaches for engineering material properties, e.g., changing the chemistry by doping or forming composite structures by physical addition, properties of the $2 \mathrm{D}$ materials exhibit 
both size- and shape dependence as well [1]. Thus, controlling the growth conditions and kinetics can further customize their properties. For example, we may refer to altering the conductivity and ferromagnetic response of $\mathrm{MoS}_{2}$ by shifting from a zigzag to an armchair structure in nanoribbons [2].

CVD method-and its variants-is a versatile technique that can control the chemistry and morphology of $2 \mathrm{D}$ materials. It has several advantages with respect to other synthesis methods, such as mechanical and chemical exfoliation techniques, including high-quality synthesis of monolayer materials with minimum contamination. However, it involves coupled physical processes with different length and time scales that hinder its understanding. Furthermore, due to the complexity of the material growth problem, a subtle change in any of the control parameters can obstruct the synthesis process. For example, a slight variation of the Mo:S ratio along the CVD reactor leads to different growth morphologies [3]. Also, a change in the growth direction from in-plane to normal to the substrate's plane upon changing the substrate's location has been reported triggered by the gradient of the precursor concentration $[4,5]$. Different 2D materials have already been synthesized using the CVD technique by trial-and-error experimentation. However, the main obstacle in their industrial application is the controllable and reproducible synthesis.

Experimental setups are commonly expensive, inflexible, and lack the versatility for changing and investigating the effect of different growth parameters and physics. Theoretical and computational methods, however, allow separating different growth parameters and physics. An industrially applicable model needs to: (i) capture the growth conditions/morphology/ property relationship, (ii) have a high-fidelity concerning experimental observations, and (iii) be computationally efficient. Furthermore, high-throughput computational simulations of such growth models combined with emerging machine learning technologies provide the opportunity for synthesis-by-design of new layered materials. Recent developments in modeling 2D materials and their growth processes are reviewed in Ref. [6], and a perspective on their controlled synthesis is given in Ref. [7].

Isolated modeling techniques have been used to investigate the growth of 2D materials [8-12]. Molecular dynamics (MD) simulations based on empirical $[13,14]$ and reactive [15-17] potentials are utilized to study the growth of these monolayered materials. Ab initio MD simulations are utilized to study nucleation mechanisms [18]. High-throughput DFT simulations have also been used to determine the formation energy of various 2D materials [19], and Phase-Field (PF) simulations are utilized to investigate different competing mechanisms governing their morphology [20-22]. Most of these studies focus on a particular aspect of growth within a limited length and time scale.

Here, we developed a multiscale/multiphysics model for the synthesis of CVD-grown monolayer materials, connecting macroscale growth parameters with the mesoscale morphology of the as-grown material. The proposed model establishes a direct connection between experimentally controllable growth parameters and the final morphology of the synthesized 2D materials. Thus, it provides an alternative to costly and timeconsuming experimental investigations.

\section{Macro- to mesoscale model of CVD growth}

Several attempts have been made to capture the various physics involved in the growth of monolayer materials across different length- and time scales. A coupled MD and finite element model was used to study thermal transport in large-area $\mathrm{MoS}_{2}$ monolayers [23]. The multiscale Kinetic Monte Carlo (KMC) model was used to study the epitaxial growth kinetics of graphene [24]. A combination of DFT, KMC, and continuum models has also been used to understand the growth kinetics of $2 \mathrm{D}$ materials [25]. A coupled DFT and continuum model has been developed to study the growth of multilayer 2D materials [26]. However, a model connecting experimentally controllable parameters to final morphology and characteristics of the as-grown monolayers is still missing.

We developed a hierarchical multiscale and multiphysics model connecting the macroscale heat and mass transport in the CVD chamber with the mesoscale growth of the 2D materials. Our model at the macroscale captures the heat transfer (conduction and convection), the flow of precursor gases, flowassisted diffusion of precursors, substrate rotation, and buoyancy forces. At mesoscale, the proposed model considers the effect of orientation-dependent edge energies, deposition rate, edge diffusion, and temperature on the growth morphology of the synthesized monolayer materials.

\section{Macroscale model of heat and mass transport}

The Navier-Stokes equation models the mass transfer in the growth chamber driven by the pressure difference and buoyancy forces,

$\rho \frac{\partial \mathbf{v}}{\partial t}+\rho(\mathbf{v} \cdot \nabla) \mathbf{v}=\nabla \cdot\left[-p \mathbf{I}+\mu\left(\nabla \mathbf{v}+(\nabla \mathbf{v})^{T}\right)\right]+\left(\rho-\rho_{r e f}\right) \mathbf{g}$.

Here, $\rho$ is the mass density of the gas mixture, $\mathbf{v}$ the velocity field, $p$ the pressure, I the unit tensor, $\mu$ the dynamic viscosity, $\mathbf{g}$ the gravitational acceleration, and superscript ${ }^{T}$ the transpose matrix. The dependable variables in this equation are pressure, $p$, and velocity field, $\mathbf{v}$.

The continuity equation, withholding the conservation of mass, is

$$
\rho \nabla \cdot \mathbf{v}=0 .
$$


In this study, we assumed $\rho$ to be a function of temperature only, as the concentration of the precursors is low in the gas phase. Flow-assisted diffusion is governed by,

$$
\frac{\partial c_{i}}{\partial t}+\nabla \cdot \mathbf{J}_{i}+\mathbf{v} \cdot \nabla c_{i}=R_{i}
$$

where,

$$
\mathbf{J}_{i}=-D_{i} \nabla c_{i}
$$

Here, $c_{i}$ is the concentration of the $i$ th species in $\mathrm{mol} / \mathrm{m}^{3}$, $D_{i}$ is the diffusion flux for the $i$ th component in $\mathrm{m}^{2} / \mathrm{s}, R_{i}$ is the source term for the $i$ th precursor in $\mathrm{mol} / \mathrm{m}^{2} \mathrm{~s}, \mathbf{v}$ is the mass averaged velocity vector in $\mathrm{m} / \mathrm{s}$, and $J_{i}$ is the diffusive flux vector in $\mathrm{mol} / \mathrm{m}^{2} \mathrm{~s}$.

The equations for the conservation of energy governing the convection and conduction heat transfer are

$$
F=\int_{V} \epsilon\left[f\left(\phi_{i}\right)-\lambda u g\left(\phi_{i}\right)+\frac{1}{2} \sum_{i} W\left(\theta_{i}\right)^{2}\left(\nabla \phi_{i}\right)^{2}\right] \mathrm{d} V
$$

where the subscript $i$ of $\phi_{i}$ denotes the islands with different orientations, $\epsilon$ is an energy scale parameter, $\lambda$ is a coupling coefficient between $\phi_{i}$ and $u ; f\left(\phi_{i}\right)=\sum_{i}\left(\phi_{i}^{2}-1\right)^{2}+$ $\alpha \sum_{i \neq j}\left(\phi_{i}+1\right)^{2}\left(\phi_{j}+1\right)^{2}$ is an energy functional with $\alpha$ being a positive constant to ensure the correct equilibrium values of $\phi_{i}$ and avoid the coexistence of two different island orientations at the same location; $g\left(\phi_{i}\right)=\frac{\phi_{i}^{5}}{5}-\frac{2 \phi_{i}^{3}}{3}+\phi_{i}$ is a monotonous interpolation function; $W\left(\theta_{i}\right)=W_{0} a\left(\theta_{i}\right)$ is the orientationdependent interface thickness where $\theta_{i}=\arctan \left(\frac{\partial_{y} \phi_{i}}{\partial_{x} \phi_{i}}\right)$ is the local surface orientation angle, $W_{0}=12 \mu \mathrm{m}$ is a reference interface thickness, and $a\left(\theta_{i}\right)$ describes the anisotropy of interface thickness.

Assuming a linear relationship between the thermodynamic driving forces and the rate of change of variables, the governing kinetic equations are

$$
\frac{1}{L\left(\theta_{i}\right) \epsilon} \frac{\partial \phi_{i}}{\partial t}=\frac{-1}{\epsilon} \frac{\delta F}{\delta \phi_{i}}=-f^{\prime}\left(\phi_{i}\right)+\lambda u g^{\prime}\left(\phi_{i}\right)+\nabla \cdot\left[W\left(\theta_{i}\right)^{2} \nabla \phi_{i}\right]-\nabla \cdot\left[W\left(\theta_{i}\right) \cdot W^{\prime}\left(\theta_{i}\right) \frac{\partial \theta_{i}}{\partial\left(\nabla \phi_{i}\right)}\right]
$$

$$
\rho C_{p} \frac{\partial T}{\partial t}+\rho C_{\mathbf{p}} \mathbf{v} \cdot \nabla T+\nabla \cdot \mathbf{q}=Q
$$

and

$$
\mathbf{q}=-k \nabla T .
$$

Here $C_{\mathrm{p}}$ is the specific heat of the gas mixture, $T$ the temperature, and $k$ the thermal conductivity.

\section{Mesoscale model of the growth}

We pursued a PF approach to model the morphology evolution of 2D materials. We made a set of assumptions to develop a computationally tractable model, i.e., we neglected the gas phase and surface chemical reactions and multilayer growth. We consider that the growth is realized by the continuous deposition of precursors from the gas phase to form the crystalline monolayers. Therefore, two sets of PF variables are used to describe the microstructure evolution of the monolayer during its growth. The first set is the non-conserved order parameter $\phi$ to distinguish the substrate $(\phi=-1)$ and a crystalline island with different orientations $(\phi=1)$. The second set is the conserved order parameter representing the reduced saturation field $u=\left(c-c_{\text {eq }}\right) / c_{\text {eq }}$ to describe concentration distribution. Here $c$ is precursor concentration, and $c_{\mathrm{eq}}$ is its equilibrium value at given temperature and pressure. The total free energy $F$, as a function of these PF variables, is

$$
\frac{\partial u}{\partial t}=\nabla \cdot\left[D\left(\phi_{i}\right) \nabla u\right]+\frac{1-h\left(\left\{\phi_{i}\right\}\right)}{2} F-\frac{1}{2} \sum_{i} \frac{\partial \phi_{i}}{\partial t},
$$

where $L\left(\theta_{i}\right)$ is an anisotropic kinetic coefficient related to interface mobility, $D\left(\phi_{i}\right)=\frac{1-h\left(\left\{\phi_{i}\right\}\right)}{2} D_{S}+\left[1-h\left(\left\{\phi_{i}\right\}\right)^{2}\right] D_{e}$ is the overall diffusivity, including the contribution from both the substrate diffusivity $D_{\mathrm{s}}$ and edge diffusivity $D_{\mathrm{e}}$, with $h\left(\left\{\phi_{i}\right\}\right)=-1+\sum_{i}\left(1+\phi_{i}\right)$; and $F$ is the deposition rate. The nucleation of $2 \mathrm{D}$ materials is realized by introducing random circular nucleus seeds. The anisotropic kinetic coefficient $L\left(\theta_{i}\right)$, similar to the anisotropic interface thickness $W\left(\theta_{i}\right)$, has the form of $L\left(\theta_{i}\right)=L_{0} a_{\mathrm{L}}\left(\theta_{i}\right)$ where $L_{0}=4.0 E 4 \mathrm{~m}^{2} / \mathrm{J}$ s. is a reference interface kinetic coefficient while $a_{\mathrm{L}}\left(\theta_{i}\right)$ describes the anisotropy of interface mobility. Considering the different energetics and kinetic properties of the armchair, zigzag and antenna edges of $\mathrm{WSe}_{2}$, the interfacial thickness anisotropy $a\left(\theta_{i}\right)$ takes the form of

$$
\begin{aligned}
a\left(\theta_{i}\right)= & a_{0}\left\{1+a_{1} \sqrt{f_{0}+\cos ^{2}\left[\frac{3}{2}\left(\theta_{i}-\theta_{i}^{0}+\frac{\pi}{2}\right)\right]}\right. \\
& \left.+a_{2} \sqrt{f_{0}+\sin ^{2}\left[\frac{3}{2}\left(\theta_{i}-\theta_{i}^{0}+\frac{\pi}{2}\right)\right]}\right\}
\end{aligned}
$$

The fitting coefficients $a_{0}, a_{1}, a_{2}$ and $f_{0}$ in Eq. (10) are fitted to align with the calculated anisotropic edge energies of $\mathrm{WSe}_{2}$ under Se-rich conditions [27], with $a_{0}=0.2653, a_{1}=2.0$, $a_{2}=1.073$ and $f_{0}=0.0001$. Similarly, the interfacial mobility anisotropy $a_{\mathrm{L}}\left(\theta_{i}\right)$ has the form of 
TABLE 1: Boundary conditions for the heat and mass transfer, Eqs. (1)-(6).

\begin{tabular}{ll}
\hline \hline Navier-Stokes & \\
No-slip on walls & $\mathbf{v}=\mathbf{0}$ \\
No-slip on rotating substrate & $\mathbf{v}=\omega \times \mathbf{r}$ \\
Normal inflow velocity & $\mathbf{v}=-\mathrm{V}_{0} \cdot \mathbf{n}$ \\
Zero outlet pressure & $-p \boldsymbol{I}+\mu\left(\nabla \mathbf{v}+(\nabla \mathbf{v})^{\top}\right)-\frac{2}{3} \mu(\nabla \cdot \mathbf{v}) \mathbf{I}=0$ \\
Heat transfer & \\
Inlet & $-\mathbf{n} \cdot(-k \nabla T)=0$ \\
Outflow & $-\mathbf{n} \cdot(-k \nabla T)=0$ \\
On furnace wall & See Fig. S2 \\
Flow-assisted diffusion & $-\mathbf{n} \cdot\left(\mathbf{J}_{i}+\mathbf{v} c_{i}\right)=0$ \\
No flux at furnace walls & Constant $c_{0}$ \\
Concentration @ source & $-\mathbf{n} \cdot D_{i} \nabla c_{i}$ \\
Outflow & $\mathbf{n} \cdot\left(\mathbf{J}_{i}+\mathbf{v c} c_{i}\right)=\mathbf{n} \cdot\left(\mathbf{v} c_{0, j}\right) ; c_{0, c}=\mathrm{cte}$ \\
Inflow & \\
\hline
\end{tabular}

Here $\mathrm{r}$ is the radial distance of a point from the center of the rotating substrate and $\mathbf{N}_{i}=-D_{i} \nabla c_{i}+\mathbf{v} c_{i}$ is the flow-assisted diffusion flux

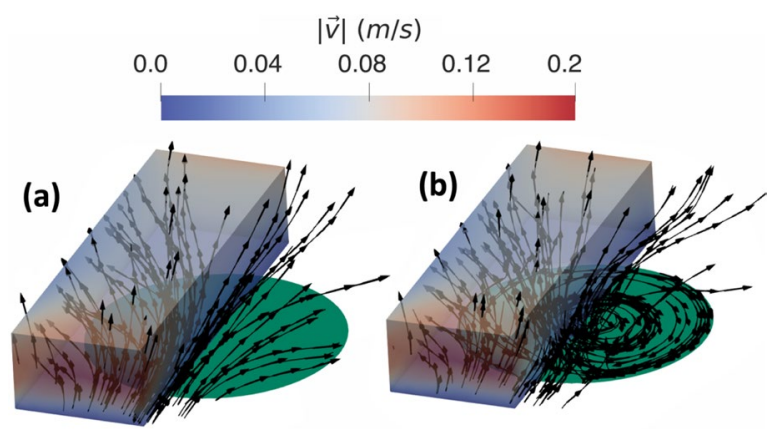

Figure 1: Effect of the substrate rotation on the velocity profile. Velocity streamlines in the volume above the substrate for stationary substrate (a) and one that is rotating at $150 \mathrm{rpm}$ (b). The colored volume shows the gas phase on the left half of the substrate, where the color map shows the velocity. The substrate is shown as green circle.
Consortium-Materials Innovation Platform (2DCC-MIP) [28]. Dimensions of this furnace and its different components are shown in Supplementary Fig. S1. Here, precursor gases are $\mathrm{W}(\mathrm{CO})_{6}$ and $\mathrm{H}_{2} \mathrm{Se}$, and the carrier gas is $\mathrm{H}_{2}$. We studied the effects of several growth parameters on the coverage and uniformity of the as-grown $\mathrm{WSe}_{2}$ monolayers, including substrate rotation, diffusion coefficients, and precursors' flow rate.

The boundary conditions used for the macroscale model of the heat and mass transport in the growth chamber are listed in Table 1 for the reference case study. The corresponding parameter values are listed in Table S1 in the Supplemental Materials. Experimentally measured wall temperature is used as the boundary condition on the furnace wall (Supplementary Fig. S2). Temperature-dependent carrier gas properties are also

$$
a_{\mathrm{L}}\left(\theta_{i}\right)=a_{L 0}\left\{1+a_{L 1} \sqrt{f_{L 0}+\cos ^{2}\left[\frac{3}{2}\left(\theta_{i}-\theta_{i}^{0}+\frac{\pi}{2}\right)\right]}+a_{L 2} \sqrt{f_{L 0}+\sin ^{2}\left[\frac{3}{2}\left(\theta_{i}-\theta_{i}^{0}+\frac{\pi}{2}\right)\right]}\right\}
$$

The fitting coefficients $\mathrm{a}_{\mathrm{L} 0}, \mathrm{a}_{\mathrm{L} 1}, \mathrm{a}_{\mathrm{L} 2}$ and $\mathrm{f}_{\mathrm{L} 0}$ in Eq. (11) should be fitted to align with the anisotropic edge mobility of $\mathrm{WSe}_{2}$ under Se-rich conditions. However, due to the lack of reliable edge mobility data, we adopt the following values in the simulation: $\mathrm{a}_{\mathrm{L} 0}=0.0025, \mathrm{a}_{\mathrm{L} 1}=1000.0, \mathrm{a}_{\mathrm{L} 2}=0$ and $\mathrm{f}_{\mathrm{L} 0}=0.000001$. The phase-field simulations are performed under zero flux boundary conditions.

\section{Application to WSe 2 growth}

We adjusted the developed model to study the synthesis of $\mathrm{WSe}_{2}$ monolayers grown by a Metal Organic Chemical Vapor Deposition (MOCVD) furnace at the 2D Crystal shown in Fig. S3, and the PF model parameters are also listed in Table S2.

\section{Effect of substrate rotation}

The velocity profile plays a significant role in determining the precursor distribution on the substrate due to the velocityassisted diffusion mechanism. Figure 1 shows the velocity profile and streamlines within the volume above the substrate. The flow velocity is high upstream, close to the inlets, decreasing over the substrate and increasing downstream closer to the outlet. The increase in the gas velocity downstream is due to the reduction in the cross-section area (see Fig. S1 in Supplementary 


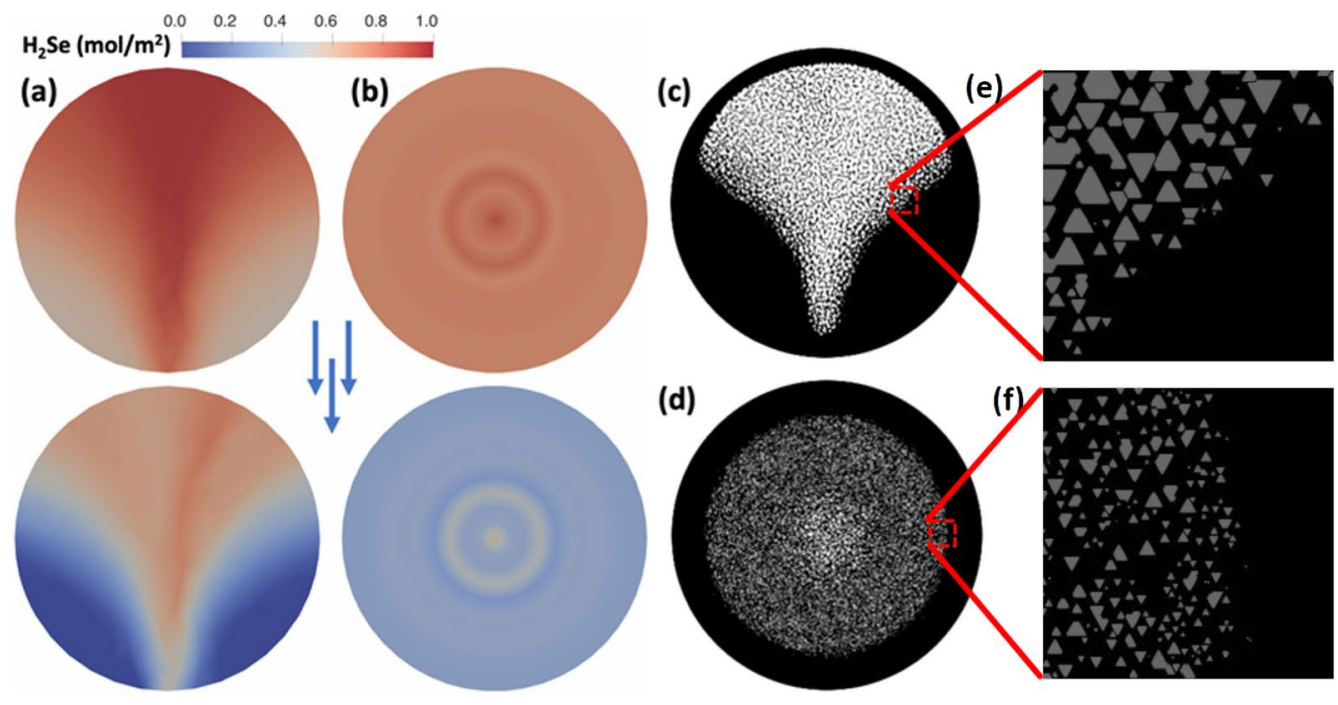

Figure 2: Effect of substrate rotation on precursor concentration. Steady-state concentration distribution of the precursors, $\mathrm{W}(\mathrm{CO})_{6}$ and $\mathrm{H}_{2} \mathrm{Se}$, for the stationary substrate (a) and one rotating at $150 \mathrm{rpm}$ (b), are shown. The PF simulation results for the corresponding growth morphology (in white color) and coverage for the stationary (c)—zoomed area shown in (e)—and rotating substrate (d)—zoomed area shown in (f)—are depicted. The bottom rows of $(\mathrm{a}-\mathrm{b})$ show the concentration distribution of $\mathrm{W}(\mathrm{CO})_{6}$ and the top rows show the $\mathrm{H}_{2} \mathrm{Se}$ concentration distribution. Arrows show the direction of the gas flow. For a stationary substrate, precursors are distributed in a conical shape following the flow streamline, resulting in uneven distribution. In contrast, precursor distribution is uniform on the rotating substrate with a higher concentration at the center.

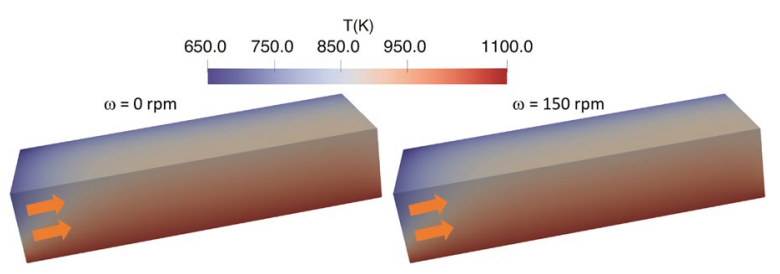

Figure 3: The temperature profile of the stationary and rotating substrate. Rotation of the substrate does not have a significant impact on the temperature of the gas phase. Arrows show the gas flow direction.
Materials) and domination of buoyancy forces as the gas is heated, passing over the susceptor.

Comparing the streamlines for the two cases of the stationary and rotating substrate, we revealed a collected set of streamlines in the latter case. Furthermore, the substrate's rotation deflected the streamlines toward the substrate, potentially increasing the effective adhesion of precursors. The streamlines, Fig. 1, also indicate a better mixing when the substrate is rotating.

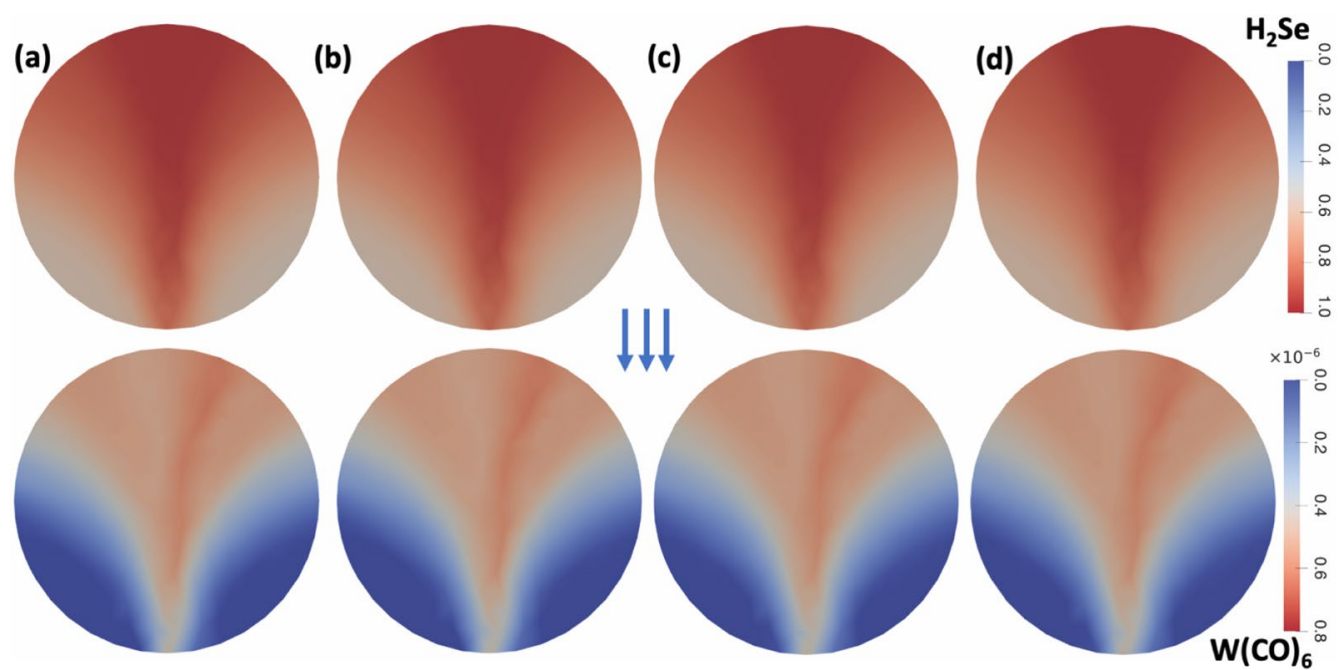

Figure 4: Precursor concentration profiles on the stationary substrate for different diffusion rates. The concentration profile of $\mathrm{H}_{2} \mathrm{Se}$ and $\mathrm{W}(\mathrm{CO})_{6}$ are shown on the top and bottom rows, respectively, for (a) $D=10^{-9} \mathrm{~m}^{2} / \mathrm{s}$, (b) $D=10^{-8} \mathrm{~m}^{2} / \mathrm{s}$, (c) $D=10^{-7} \mathrm{~m}^{2} / \mathrm{s}$, and (d) $D=10^{-6} \mathrm{~m}^{2} / \mathrm{s}$. 


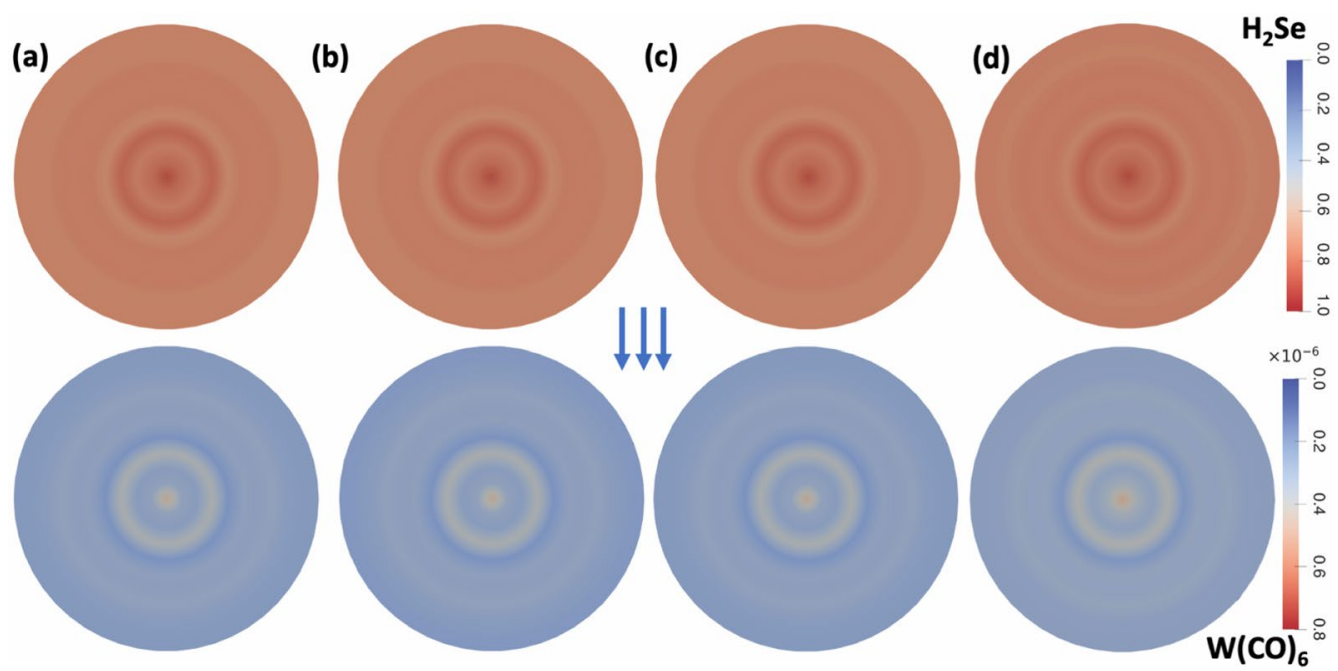

Figure 5: Precursor concentration profiles on the substrate rotating at $150 \mathrm{rpm}$ for different diffusion rates. The concentration profile of $\mathrm{H}_{2} \mathrm{Se}$ and $\mathrm{W}(\mathrm{CO})_{6}$ are shown on the top and bottom rows, respectively. (a) $D=10^{-9} \mathrm{~m}^{2} / \mathrm{s}$, (b) $D=10^{-8} \mathrm{~m}^{2} / \mathrm{s}$, (c) $D=10^{-7} \mathrm{~m}^{2} / \mathrm{s}$, and (d) $D=10^{-6} \mathrm{~m}^{2} / \mathrm{s}$.

The concentration distribution of precursors, $\mathrm{H}_{2} \mathrm{Se}$ and $\mathrm{W}(\mathrm{CO})_{6}$, and the $\mathrm{WSe}_{2}$ growth coverage over the substrate at steady-state conditions is shown in Fig. 2, where arrows indicate the flow direction. Our results revealed a more uniform precursor distribution over the substrate when the substrate is rotating and a higher concentration of precursors at the center that potentially leads to multilayer growth. In contrast, most of the precursor concentration in the case of the stationary substrate is concentrated at its front edge, leading to large concentration gradients, which promotes the formation of out-of-plane growth and agglomeration by Mullins-Sekerka instability [4, 5]. Substrate's rotation also enhances the intermixing of precursors, providing a more uniform W: Se ratio and a more consistent final synthesis morphology over the substrate, as shown in our PF simulations in Fig. 2c-d.

Reduction in the concentration of precursors downstream can be explained by the domination of buoyancy forces as the carrier gas warms up when it passes over the heated substrate. The concentration gradient of $\mathrm{W}(\mathrm{CO})_{6}$ is higher than the $\mathrm{H}_{2} \mathrm{Se}$, which is due to the relative position of the inlets concerning the substrate. The $\mathrm{H}_{2} \mathrm{Se}$ inlet is at a higher height compared to $\mathrm{W}(\mathrm{CO})_{6}$. Thus, diffusion of $\mathrm{H}_{2}$ Se plays a more dominant role than flow-assisted diffusion, resulting in its more uniform distribution. The temperature profile of the gas close to the substrate is shown in Fig. 3, indicating no significant difference in steady-state temperature profile between the two stationary and rotating substrates.

\section{Effect of diffusion coefficient}

One of the key growth parameters is the diffusion coefficient of precursors within the gas phase. Although it cannot be directly controlled, it is a strong function of temperature and pressure. Here, we performed a systematic study to understand the role
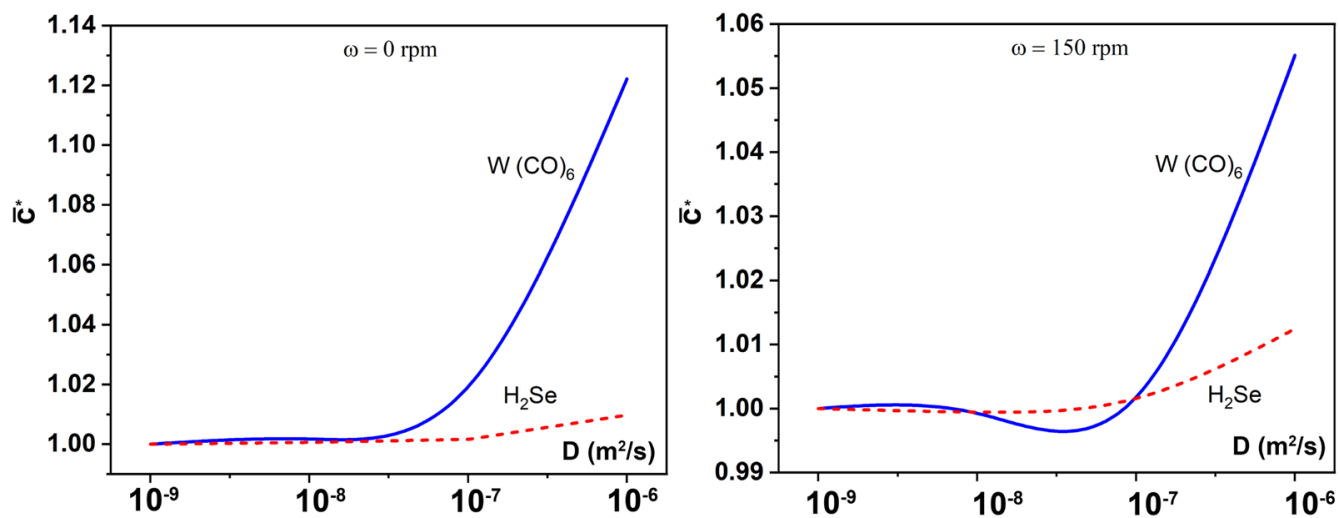

Figure 6: Effect of diffusion coefficient on the average precursor concentration. Normalized average concentration, $\bar{c}^{*}$, of the two precursors deposited on the substrate is plotted for four diffusion coefficients. Our results indicate a direct correlation between $\bar{c}^{*}$ and gas phase diffusion coefficients for both cases of stationary and rotating substrates. 

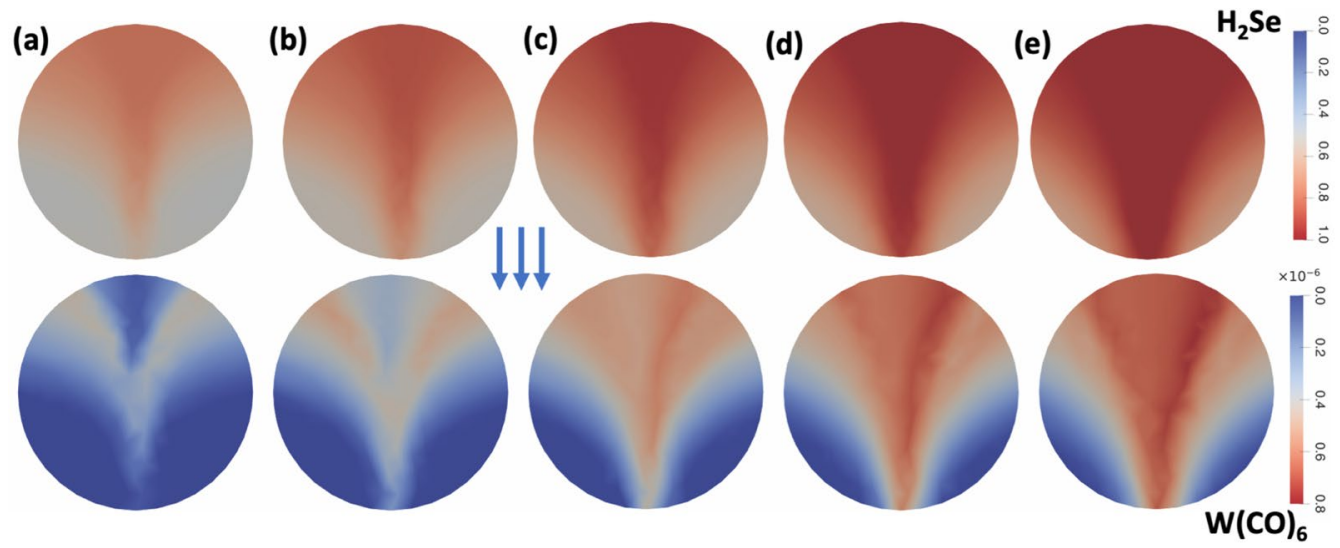

Figure 7: Precursor concentration profiles on the stationary substrate for different inlet velocities. The concentration profile of $\mathrm{H}_{2} \mathrm{Se}$ and W(CO) 6 are shown on the top and bottom rows, respectively, for nominal inlet velocity (c), $20 \%$ and $10 \%$ reduction (a-b), and $10 \%$ and $20 \%$ increase in nominal inlet velocity (d-e). Arrows show the direction of the gas flow.
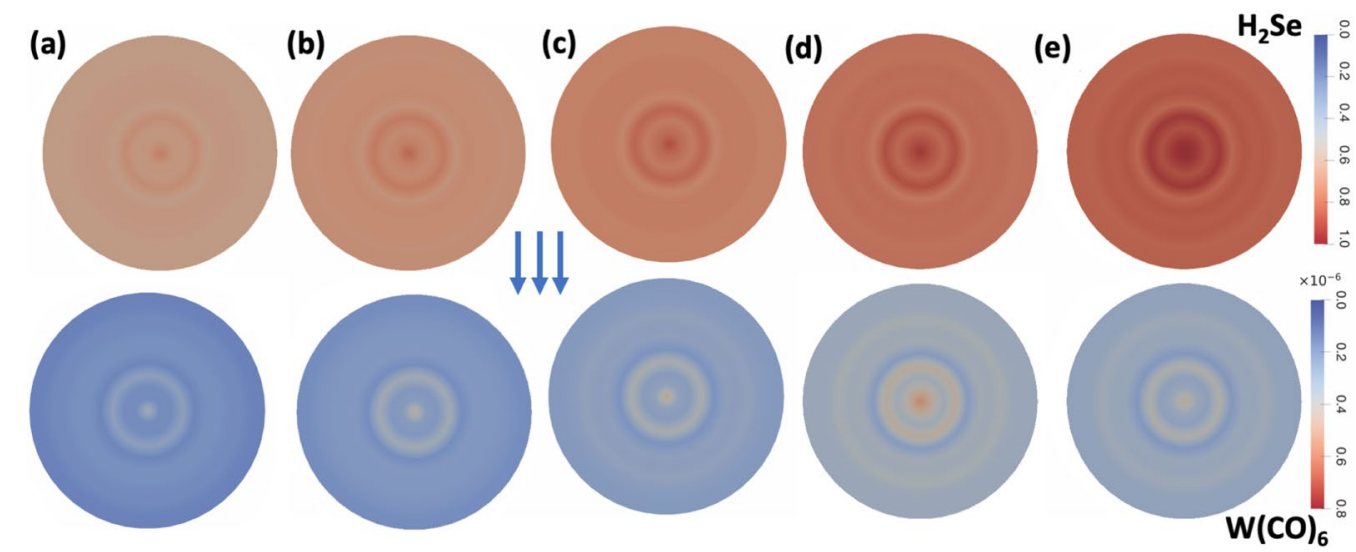

Figure 8: Precursor concentration profiles on the substrate for the substrate rotating at $150 \mathrm{rpm}$ for different inlet velocities. The concentration profile of $\mathrm{H}_{2} \mathrm{Se}$ and $\mathrm{W}(\mathrm{CO})_{6}$ are shown on the top and bottom rows, respectively, for nominal inlet velocity (c), $20 \%$ and $10 \%$ reduction (a-b), and $10 \%$ and $20 \%$ increase in nominal inlet velocity ( $d-e)$. Arrows show the direction of the gas flow.

of the diffusion coefficient. We considered four cases with diffusion coefficients ranging from $10^{-9}$ to $10^{-6} \mathrm{~m}^{2} / \mathrm{s}$. The precursor concentration profiles on the substrate are shown in Figs. 4 and 5 for the stationary and rotating substrates, respectively. The corresponding PF simulations showing the growth coverage are shown in Supplementary Fig. S4.

Our results indicate that the conical deposition region of the stationary substrate widens as the diffusion coefficient increases. We also did not see a significant change in the profile of precursor concentration on the rotating substrate upon variation in the diffusion coefficient. Figure 6 shows the average concentration of precursors normalized by their corresponding mean values for the reference case $D=10^{-9} \mathrm{~m}^{2} / \mathrm{s}$, $\bar{c}^{*}=\bar{c} /\left.\bar{c}\right|_{D=10^{-9}}$. Furthermore, the average concentration of precursors deposited on the stationary substrate increases by increasing diffusion coefficient, Fig. 6. Comparing the normalized mean values for the stationary and rotating substrates, we concluded that the mean value of precursor species on the stationary substrate is more sensitive to the diffusion coefficient. Furthermore, the mean value of $\mathrm{H}_{2} \mathrm{Se}$ concentration on the substrate is less sensitive to the diffusion coefficient than the one for $\mathrm{W}(\mathrm{CO})_{6}$. Thus, the position of inlets relative to the substrate plays a significant role in reducing the sensitivity of the final growth morphology to unavoidable variations in the growth conditions.

\section{Effect of inlet velocity}

The inlet velocity of precursors and carrier gas plays a major role in determining the concentration profile of precursors on the substrate via the flow-assisted diffusion process. Our simulation results are shown in Figs. 7 and 8, and corresponding growth morphologies are depicted in Supplemental Fig. S5. We revealed a higher sensitivity of the stationary substrate 

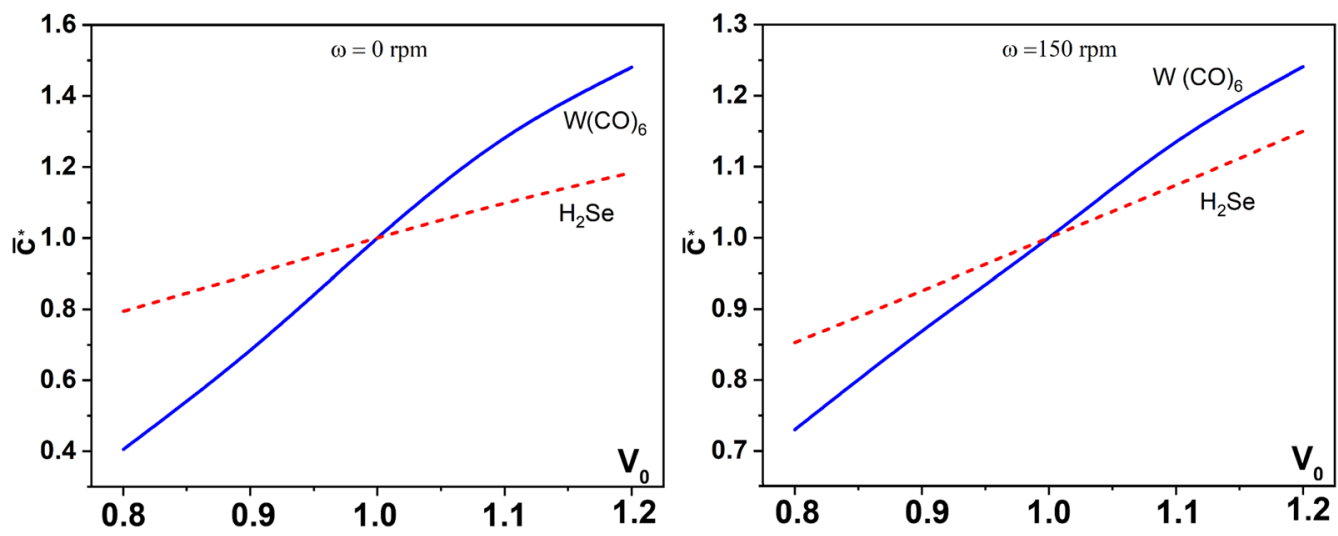

Figure 9: Effect of inlet velocity on the average precursor concentration. Normalized average concentration, $\bar{c}^{*}$, of the two precursors deposited on the substrate is plotted for five different cases, where the inlet velocity varied from $-20 \%$ to $+20 \%$ of the nominal inlet velocity $\mathrm{V}_{0}$. Our results indicate a direct correlation between $\bar{c}^{*}$ and inlet velocities for both cases of stationary and rotating substrates. Although, this relation is not monotonous in the case of a rotating substrate.

to variations of the inlet velocity. Furthermore, reduction of the inlet velocity has a more profound effect on the distribution of the $\mathrm{W}(\mathrm{CO})_{6}$, where deposition on the front edge of the substrate drastically reduces. It is due to the positioning of the substrate at the end of a ramp and the relative location of the $\mathrm{W}(\mathrm{CO})_{6}$ inlet with respect to the substrate, i.e., a lower distance with the plane of the substrate. As a result, the inlet flow of $\mathrm{W}(\mathrm{CO})_{6}$ and carrier gas will deflect upon hitting the ramp, forming a boundary layer. The thickness of this boundary layer is an inverse function of the inlet and carrier gas velocity. Thus, reducing the inlet gas velocity results in a thicker boundary layer, which increases the mean free path of the precursor atoms for reaching the substrate, reducing the concentration of $\mathrm{W}(\mathrm{CO})_{6}$.

In contrast to $\mathrm{W}(\mathrm{CO})_{6}$, the $\mathrm{H}_{2}$ Se inlet is located at a higher height. Thus, no boundary layer forms, and reduction in the $\mathrm{H}_{2} \mathrm{Se}$ inlet velocity directly reduces the flow-assisted diffusion of precursors and a more uniform concentration distribution of the precursors on the substrate. In the case of a rotating substrate, the formed boundary layer results in lower concentration of $\mathrm{W}(\mathrm{CO})_{6}$ on the outer section of the substrate, which may hinder the growth of $2 \mathrm{D}$ materials in this section.

The average precursor concentration as a function of the inlet velocity normalized by the one for the reference simulations (see Table S1 in the Supplemental Materials) is shown in Fig. 9. Our results indicate a direct correlation between the average precursor concentration and the inlet velocity for a stationary substrate and higher sensitivity of the $\mathrm{W}(\mathrm{CO})_{6}$ to the inlet velocity variations. Also, the variation of the precursor concentration is larger for reduction of the inlet velocity than its increase. The average precursor concentration is proportional to the inlet velocity but less sensitive to variations when the substrate is rotating.

\section{Conclusion}

We developed a coupled fluid mechanics/PF multiscale model for the growth of monolayer materials, connecting macroscale synthesis parameters to the mesoscale morphology. The model considers various physics, including heat and mass transfer in the CVD chamber, flow-assisted diffusion of precursors, buoyancy forces, surface and edge diffusion, orientation-dependent edge energies, deposition rate, temperature, and pressure. It is unique in its versatility for modeling CVD growth of various $2 \mathrm{D}$ materials and establishing a correlation between experimentally controllable parameters, such as precursors' and carrier gas flow rates, with mesoscale morphology of the as-grown materials.

Our results indicate a more uniform growth upon rotating the substrate, with a higher chance of multilayer growth at the center. We revealed a higher concentration of precursors on the front edge of the stationary substrate, which may lead to the formation of vertical nanofins or agglomerates due to Mullins-Sekerka instability and the Volmer-Weber mechanism. The precursor distribution on the stationary substrate is more sensitive to the buoyancy forces, diffusion coefficient, and inlet velocity. It also reduces downstream, disrupting the growth of 2D materials. We revealed a lower growth coverage on the stationary substrate and a higher growth density on a rotating substrate upon increasing the diffusion coefficient. Our results also indicate higher sensitivity of the average precursor concentration to the diffusion coefficient for the stationary substrate than the rotating substrate. We further revealed a higher deposition rate and growth coverage upon increasing the inlet velocity by increasing the velocity-assisted diffusion of precursors. The average precursor concentration is directly proportional to the inlet velocity and monotonously varies by the inlet velocity. Furthermore, the deposited precursor concentration is less sensitive to the inlet velocity for a rotating substrate. 
We established correlations between different experimentally controllable parameters and the coverage and synthesis quality of 2D materials. We analyzed the sensitivity of the final growth quality to experimentally controllable growth parameters and proposed several ways for reducing this sensitivity. In the future, we will couple our model with the atomistic simulations calculating the orientation-dependent edge energies as a function of gas phase chemistry. This capability will allow capturing the variation in the growth morphology as a function of precursor concentration ratios.

\section{Methods}

The macro- and mesoscale simulations are coupled via the precursor concentration distribution over the substrate. The solution domain of the macroscale model is the entire growth chamber, while it is limited to the substrate for the PF simulations. The macroscale model of the flow is solved using the finite volume method, and the PF model is solved using the finite difference technique. We have neglected the depletion of precursor on the substrate due to the island growth. Because the growth is localized on the surface and the as-grown 2D materials are only a single atom thick and thus have a subtle effect on the macroscopic transport phenomena. We further assume the deposition process from supersaturated precursors vapor.

The macroscale model is meshed using free tetrahedral elements. We refined the mesh on the substrate, the inlets, the outlet, and the adjacent volumes to improve simulations' accuracy and get mesh-independent results. We utilized the Galerkin least-squares method to discretize the governing coupled system of equations. We choose a test function that stabilizes the hyperbolic terms and the pressure term in the transport equations. Furthermore, we used the Galerkin formulations to conserve momentum, mass, and energy. Finally, we utilized the damped Newton method and time-stepping techniques with automatic time-stepping and automatic polynomial orders to resolve the velocity and pressure fields. The mesoscale PF simulations are performed using our in-house finite difference code. A minimum of five elements per interface width is considered to have mesh-independent results.

\section{Acknowledgments}

This project is partly supported by the University of Alabama, the NSF-CAREER under the NSF cooperative agreement CBET-2042683, and 2D Crystal Consortium - Material Innovation Platform (2DCC-MIP) under NSF cooperative agreement DMR-1539916 and the I/UCRC Center for Atomically Thin Multifunctional Coatings (ATOMIC) Seed Project SP00117. The authors also thank Dr. Joshua A. Robinson, Dr. Joan
Redwing, Dr. Tanushree Holme Choudhury, and Mr. Haoyue Zhu for the fruitful discussions.

\section{Data availability}

All data generated or analyzed during this study are included in this published article and its supplementary information files.

\section{Declarations}

Conflict of interest On behalf of all authors, the corresponding author states that there is no conflict of interest.

\section{Supplementary Information}

The online version contains supplementary material available at https://doi.org/10.1557/s43578-021-00384-2.

\section{Open Access}

This article is licensed under a Creative Commons Attribution 4.0 International License, which permits use, sharing, adaptation, distribution and reproduction in any medium or format, as long as you give appropriate credit to the original author(s) and the source, provide a link to the Creative Commons licence, and indicate if changes were made. The images or other third party material in this article are included in the article's Creative Commons licence, unless indicated otherwise in a credit line to the material. If material is not included in the article's Creative Commons licence and your intended use is not permitted by statutory regulation or exceeds the permitted use, you will need to obtain permission directly from the copyright holder. To view a copy of this licence, visit http://creativecommons.org/ licenses/by/4.0/.

\section{References}

1. W. Hu, L. Lin, C. Yang, J. Dai, J. Yang, Edge-modified phosphorene nanoflake heterojunctions as highly efficient solar cells. Nano Lett. 16, 1675-1682 (2016). https://doi.org/10. 1021/acs.nanolett.5b04593

2. Y. Li, Z. Zhou, S. Zhang, Z. Chen, $\mathrm{MoS}_{2}$ nanoribbons: high stability and unusual electronic and magnetic properties. J. Am. Chem. Soc. 130, 16739-16744 (2008). https://doi.org/10. 1021/ja805545x

3. D. Cao, T. Shen, P. Liang, X. Chen, H. Shu, Role of chemical potential in flake shape and edge properties of monolayer $\mathrm{MoS}_{2}$. J. Phys. Chem. C. 119, 4294-4301 (2015). https://doi. org/10.1021/jp5097713

4. F. Zhang, K. Momeni, M.A. AlSaud, A. Azizi, M.F. Hainey Jr, J.M. Redwing, et al., Controlled synthesis of 2D transition metal dichalcogenides: from vertical to planar $\mathrm{MoS}_{2}$. 2D Mater. 4, 025029 (2017). 
5. R.A. Vilá, K. Momeni, Q. Wang, B.M. Bersch, N. Lu, M.J. Kim, et al., Bottom-up synthesis of vertically oriented two-dimensional materials. 2D Mater. 3, 041003 (2016).

6. K. Momeni, Y. Ji, Y. Wang, S. Paul, S. Neshani, D.E. Yilmaz et al., Multiscale computational understanding and growth of 2D materials: a review. NPJ Comput. Mater. 6, 1-18 (2020). https://doi.org/10.1038/s41524-020-0280-2

7. J. Zhang, F. Wang, V.B. Shenoy, M. Tang, J. Lou, Towards controlled synthesis of $2 \mathrm{D}$ crystals by chemical vapor deposition (CVD). Mater. Today 40, 132-139 (2020)

8. M. Seymour, N. Provatas, Structural phase field crystal approach for modeling graphene and other two-dimensional structures. Phys. Rev. B 93, 035447 (2016). https://doi.org/10.1103/PhysR evB.93.035447

9. V.I. Artyukhov, Y. Liu, B.I. Yakobson, Equilibrium at the edge and atomistic mechanisms of graphene growth. Proc. Natl. Acad. Sci. USA 109, 15136-15140 (2012). https://doi.org/10.1073/pnas. 1207519109

10. E. Meca, J. Lowengrub, H. Kim, C. Mattevi, V.B. Shenoy, Epitaxial graphene growth and shape dynamics on copper: phase-field modeling and experiments. Nano Lett. 13, 5692-5697 (2013). https://doi.org/10.1021/nl4033928

11. E. Meca, V.B. Shenoy, J. Lowengrub, Phase-field modeling of two-dimensional crystal growth with anisotropic diffusion. Phys. Rev. E. 88, 052409 (2013). https://doi.org/10.1103/PhysRevE.88. 052409

12. V.I. Artyukhov, Z. Hu, Z. Zhang, B.I. Yakobson, Topochemistry of Bowtie- and star-shaped metal dichalcogenide nanoisland formation. Nano Lett. 16, 3696-3702 (2016). https://doi.org/10. 1021/acs.nanolett.6b00986

13. A. Kandemir, H. Yapicioglu, A. Kinaci, T. Çağın, C. Sevik, Thermal transport properties of $\mathrm{MoS}_{2}$ and MoSe2 monolayers. Nanotechnology 27, 055703 (2016). https://doi.org/10.1088/ 0957-4484/27/5/055703

14. P. Norouzzadeh, D.J. Singh, Thermal conductivity of singlelayer WSe2 by a Stillinger-Weber potential. Nanotechnology $\mathbf{2 8}$, 075708 (2017). https://doi.org/10.1088/1361-6528/aa55e1

15. X. Huang, H. Yang, A.C.T. van Duin, K.J. Hsia, S. Zhang, Chemomechanics control of tearing paths in graphene. Phys. Rev. B. 85, 195453 (2012)

16. S. Paul, K. Momeni, Mechanochemistry of stable diamane and atomically thin diamond films synthesis from Bi- and multilayer graphene: a computational study. J. Phys. Chem. C. 123, 15751-15760 (2019). https://doi.org/10.1021/acs.jpcc.9b02149

17. S. Paul, K. Momeni, V.I. Levitas, Shear-induced diamondization of multilayer graphene structures: a computational study. Carbon 167, 140-147 (2020). https://doi.org/10.1016/j.carbon.2020.05. 038
18. D.G. Sangiovanni, G.K. Gueorguiev, A. Kakanakova-Georgieva, $\mathrm{Ab}$ initio molecular dynamics of atomic-scale surface reactions: insights into metal organic chemical vapor deposition of AlN on graphene. Phys. Chem. Chem. Phys. 20, 17751-17761 (2018). https://doi.org/10.1039/C8CP02786B

19. N. Mounet, M. Gibertini, P. Schwaller, D. Campi, A. Merkys, A. Marrazzo et al., Two-dimensional materials from highthroughput computational exfoliation of experimentally known compounds. Nat. Nanotechnol. 13, 246-252 (2018). https://doi. org/10.1038/s41565-017-0035-5

20. J. Li, Z. Hu, Y. Yi, M. Yu, X. Li, J. Zhou et al., Hexagonal boron nitride growth on $\mathrm{Cu}-\mathrm{Si}$ alloy: morphologies and large domains. Small 15, 1805188 (2019). https://doi.org/10.1002/smll.20180 5188

21. J. Yanzhou, K. Momeni, L.-Q. Chen, A multiscale insight into the growth of h-BN: effect of the enclosure. 2D Mater. 8, 035033 (2021)

22. K. Momeni, Y. Ji, K. Hang, et al. Multiscale framework for simulation-guided growth of 2D materials. npj 2D Mater. Appl. 2, 27 (2018). https://doi.org/10.1038/s41699-018-0072-4

23. B. Mortazavi, R. Quey, A. Ostadhossein, A. Villani, N. Moulin, A.C.T. van Duin et al., Strong thermal transport along polycrystalline transition metal dichalcogenides revealed by multiscale modeling for $\mathrm{MoS}_{2}$. Appl. Mater. Today 7, 67-76 (2017). https:// doi.org/10.1016/j.apmt.2017.02.005

24. H. Jiang, Z. Hou, Large-scale epitaxial growth kinetics of graphene: a kinetic Monte Carlo study. J. Chem. Phys. 143, 084109 (2015). https://doi.org/10.1063/1.4929471

25. H. Jiang, Z. Hou, Mechanisms beyond energetics revealed by multiscale kinetic modeling of 2D-material growth and nanocatalysis. WIREs Comput. Mol. Sci. (2021). https://doi.org/10. 1002/wcms.1524

26. H. Ye, J. Zhou, D. Er, C.C. Price, Z. Yu, Y. Liu et al., Toward a mechanistic understanding of vertical growth of van der waals stacked 2D materials: a multiscale model and experiments. ACS Nano 11, 12780-12788 (2017). https://doi.org/10.1021/acsnano 7b07604

27. N. Nayir, Y. Wang, S. Shabnam, D.R. Hickey, L. Miao, X. Zhang et al., Modeling for structural engineering and synthesis of two-dimensional $\mathrm{WSe}_{2}$ using a newly developed ReaxFF reactive force field. J. Phys. Chem. C. (2020). https://doi.org/10.1021/acs. jpcc.0c09155

28. M. Chubarov, T.H. Choudhury, D.R. Hickey, S. Bachu, T. Zhang, A. Sebastian et al., Wafer-scale epitaxial growth of unidirectional WS2 monolayers on sapphire. ACS Nano 15, 2532-2541 (2021). https://doi.org/10.1021/acsnano.0c06750 